(C) 1985 IEEE. Personal use of this material is permitted. However, permission to reprint/republish this material for advertising or promotional purposes or for creating new collective works for resale or redistribution to servers

\title{
STATISTICS OF DIPOLE STEERING IN THE TEVATRON
}

\author{
M. J. Syphers \\ Fermi National Accelerator Laboratory* \\ P. 0. Box 500
}

Batavia, IL 60510

\section{Abstract}

Methods of calculating the expected rms orbit distortion and the expected peak orbit distortion for a large FODO synchrotron are described. An expression for the expected rms correction element dipole strength necessary to correct the closed orbit of a large FODO synchrotron is derived. The uncorrected closed orbit of the FNAL Tevatron accelerator is determined from beam posttion data and operational settings of the correction element dipole magnets. The uncorrected orbit and correction element dipole strengths are compared to the results of the Tevatron design study. Use of these expressions for SSC lattices is discussed.

\section{Closed Orbit Distortions}

From the basic theory of the Alternating Gradient Synchrotron (AGS) ${ }^{1}$, a single dipole field error at longitudinal coordinate so will create adistortion of the closed orbit elsewhere in the machine given by

$$
x(s)=\frac{\theta_{0} \sqrt{\beta(s) \beta_{0}}}{2 \sin \pi \nu} \cos \left[\psi(s)-\psi\left(s_{0}\right)-\pi \nu\right] .
$$

Here, $s$ is the longitudinal position around the accelerator, $x$ is the transverse displacement from the design orbit, 3 and $\psi$ are the standard Courant-Snyder betatron amplitude and phase functions, $\nu$ is the machine tune (number of betatron oscillations in one revolution), and $\theta_{0}$ is the angular deflection produced by, the dipole Field error $[\theta=(\Delta B \ell) /(B \rho)$, where $\ell=$ length of the region over which the error occurs, $B \rho=$ magnetic rigidity].

The two major sources of dipole field errors in the large AGS are, of course, the errors in the fields of the main dipole bending magnets and the accuracy of the placement of the main quadrupole magnets. The bending magnets can have field errors contributed by non-uniform magnet lengths $(\Delta B / B=\Delta L / L$ creates deviation in horfzontal bend strength) and by roll errors (i.e., skewness). Roll errors are of special concern in the Tevatron where the magnet coils may move about during cooldown, warm-up, magnet transport and installation, etc. Roll errors greatly affect the vertical orbit $(\triangle B / B=\sin \phi, \phi=$ roll angle). Of course, any uncertainty in the field direction will contribute dipole field errors in the vertical plane.

Quadrupole magnets contribute dipole field errors due to alignment errors. If a quadrupole is displaced from its desired position by an amount d, then it will create $a$ bend field error of $\Delta B=B^{\prime} d$, where $B^{\prime}=$ the quadrupole gradient.

The uncorrected closed orbit distortion around an entire machine due to $\mathrm{N}$ dipole errors is given by

$x=\frac{\sqrt{\beta(s)}}{2 \sin \pi \nu} \sum_{i=1}^{N} \theta\left(s_{i}\right)\left[\beta\left(s_{i}\right)\right]^{\frac{L}{2}} \cos \left[\psi_{i}-\pi \nu\right]$

where $\psi_{i}=$ the betatron phase advance from point $s_{1}$ to point $s$.

*Operated by the Universities Research Association,

Inc., under contract with the U.S. Department of Energy.
By assuming that the $\mathbb{N}$ dipole field errors around the machine are uncorrelated and follow a Gaussian distribution with a typical value of $\theta_{\text {rms }}$, a typical value for the orbit distortion in the accelerator is given by ${ }^{2}$

$$
\operatorname{Xrms}(s)=\frac{\theta_{\mathrm{rms}} \sqrt{\beta(\mathrm{s})<\beta>}}{2|\sin \pi|}\left[\frac{\mathrm{N}}{2}\right]^{\frac{1}{2}} \text {. }
$$

From a Monte Carlo simulation of orbit distortions in a large FODO synchrotron, the typical value of the maximum orbit distortion is found to be about 2.2 times the expected rms orbit distortion. The probability of having a maximum distortion greater than or equal to twice the rms distortion is about 60 percent.

\section{Dipole Steering}

Assume a set of uncorrelated, randomly generated dipole field errors, obeying a Gaussian distribution, is placed about the accelerator. Periodically throughout the machine lattice (at each focusing quadrupole location, say), a dipole correction magnet is found. An expression for the expected strength of a typical dipole correction magnet necessary to correct the machine's closed orbit can be developed.

The typical steering dipole is located in the middle of a standard cell as shown in Figure 1. The dipole of interest will be the steering dipole at location 1. The orbit is assumed to be centered up to location 0 . The beam will deviate from the ideal

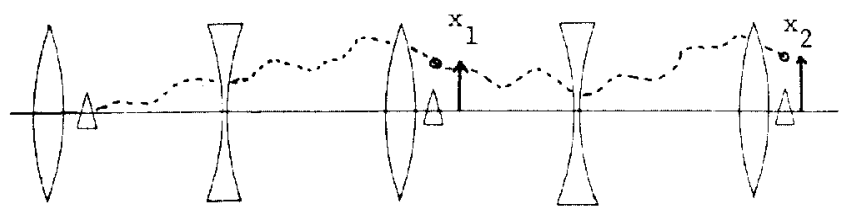

STANDARD CELL

FIGURE 1 - Standard cell used in determining expected strength of a typical steering dipole magnet.

centered closed orbit due to dipole errors $\theta_{i}$ located between 0 and 2 . To center the orbit in the region 0 to 2 the dipole at 1 is thought of as being adjusted in two steps. The first step is to steer the beam trom the value $x_{2}$ to $x=0$ at location 2 . The second step is to bring the beam position at location 1 from $x_{1}$ to zero without disturbing the orbit elsewhere in the machine using a standard "three bump" technique. Letting $\theta$ represent an angular deflection generated by a correction element dipole, these two steps can be summarized as follows:

STEP I:

$$
\theta_{1}^{\prime} \sqrt{\beta_{1} \beta_{2}} \sin \psi_{12}+x_{2}=0
$$

STEP 2:

$$
\theta_{0}^{\prime \prime} \sqrt{\beta_{0} \beta_{1}} \sin \psi_{01}+x_{1}=0
$$


and

$$
\theta_{1}^{\prime \prime}=-\theta_{0}^{\prime \prime} \sqrt{\frac{\beta_{o}}{\beta_{1}}} \frac{\sin \psi_{02}}{\sin \psi_{12}} \text { (3-bump ratio). }
$$
The total required strength of dipole $1, \Theta_{1}$, is thus
given by

$$
\theta_{1}=\theta_{1}^{\prime}+\theta_{1}^{\prime \prime}
$$

Due to dipole field errors $\theta_{i}$ in the region $s_{o}$ to $s_{2}$, the displacements at $s_{1}$ and $s_{2}$ are given by

$$
\begin{aligned}
& x_{2}=\sum_{s_{0}}^{s_{2}} \theta_{i} \sqrt{\beta_{i} \beta_{2}} \sin \psi_{i 2} \\
& x_{1}=s_{0}^{s_{1}} \theta_{i} \sqrt{\beta_{1} \beta_{1}} \sin \psi_{11} .
\end{aligned}
$$

Thus, after solving for $\Theta_{1}^{\prime}$ and $\Theta_{1}^{\prime \prime}$, individually, and realizing that these steering dipoles are at standard cell lucalions where the belatrun phase advance between steering dipoles is a constant, $\mu_{0}$, then (with a little work) the expression for $\theta_{1}$ becomes

$$
\theta_{1}=-\frac{1}{\sqrt{\beta_{0}} \sin \mu_{0}} \sum_{0}^{s_{0}} \theta_{1} \beta_{1}^{\frac{1}{2}} \sin \left(\mu_{0}-\left|\psi_{11}\right|\right) .
$$

The above expression can be considered the steering strength required at $s_{1}$ in the $m$-th accelerator of a large ensemble of accelerators $(m=1$ to $P$ ) with all accelerators identical except for the normally distributed uncorrelated random errors $\left(\theta_{i}\right)_{m}$. By squaring Eq. (4) and realizing that

$$
\left.\frac{1}{p} \sum_{m=1}^{p}\left(\theta_{i}\right)_{m}\left(\theta_{j}\right)_{m}=\delta_{i j}<\theta^{2}\right\rangle
$$

for uncorrelated $\vartheta_{i}$ 's, one finds that the rms value of $\theta_{1}$ for a large ensemble of accelerators is given by

$$
\theta_{\text {rms }}=\frac{\theta_{\mathrm{rms}}}{\operatorname{sin\mu _{0}}}\left[\frac{\langle\beta\rangle}{\beta_{0}}\right]^{\frac{1}{2}} \mathrm{~N}^{\frac{1}{2}} \text {. }
$$

Here, $N$ is the number of dipole errors within a standard cell and $\langle\beta\rangle=\left(\Sigma \beta_{i}\right) / \mathrm{N}$.

To ensure that the orbit can be centered at all locations (e.g., 90 percent probability of successful. correction at 100 locations), then the correction elements should be able to deliver a maximum deflection of more than three times this rms value.

To emphasize the dependences upon quadrupole alignment errors and dipole magnet field errors, Equations (3) and (5) may be re-written as

$$
\begin{aligned}
& \text { Xrms }=\frac{\sqrt{\beta_{0}^{<3>N_{Q}}}}{2 \sqrt{2} F|\sin \pi \nu|}\left[q^{2}+\frac{4 \pi^{2} F^{2}}{N_{B} N_{Q}} b^{2}\right]^{\frac{1}{2}} \\
& \theta_{r m s}=\sqrt{\frac{<\beta>\nabla_{Q}}{\beta_{0} \sin ^{2} \mu_{0}}} \frac{\left[q^{2}+\frac{n_{B}}{n_{Q}} \frac{4 \pi^{2} F^{2}}{N_{B}^{2}} b^{2}\right]^{\frac{1}{2}}}{F}
\end{aligned}
$$

where

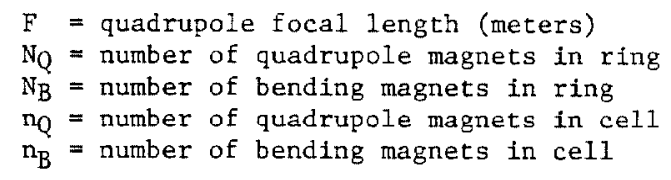

and

$$
\begin{aligned}
& q=\text { rms quadrupole displacement error (mm) } \\
& b=\text { rms dipole } \Delta B / B \text { (parts per thousand) }
\end{aligned}
$$

\section{Results for the Tevatron}

Using Tevatron parameters $\left[\beta_{0}=100 \mathrm{~m},\langle\beta\rangle=60 \mathrm{~m}\right.$, $\mathrm{F}=25 \mathrm{~m}, v=19.4, \quad \mu_{0}=70^{\circ}, \mathrm{N}_{\mathrm{R}}=220, \mathrm{~N}_{\mathrm{B}}=770$, $\left.{ }^{n_{Q}}=2, n_{B}=8\right]$, the expressions become

$$
\begin{aligned}
& \operatorname{xrms}=17 \mathrm{~mm} \quad\left[q^{2}+1 / 7 b^{2}\right]^{\frac{1}{2}} \\
& \theta_{\mathrm{rms}}=47 \mu \mathrm{rad}\left[\mathrm{q}^{2}+1 / 6 \mathrm{~b}^{2}\right]^{\frac{1}{2}} .
\end{aligned}
$$

In the Tevatron Deslgn Report ${ }^{3}$, estimates for $q$ and $b$ were made, namely $q=.5, b=1.4$. Using these numbers yields

$$
\begin{array}{ll}
\text { Xrms }=13 \mathrm{~mm} & \text { (predicted) } \\
\Theta_{\text {rms }}=32 \mu \mathrm{rad} . & \text { (predicted) }
\end{array}
$$

Thus, from the value of Xrms, one might expect the Tevatron to have a maximum orbit distortion of about $30 \mathrm{~mm}$. Likewise, the maximum steering angle which the correction dipoles would need to produce was expected to be about 100 prad. This translates to an integrated field strength of .33 Tesla-meters $(130 \mathrm{kG}-\mathrm{in}$ ) at $1 \mathrm{TeV}$. To be safe, the Tevatron correction dipole specifications were set to $.43 \mathrm{~T}-\mathrm{m}(170 \mathrm{kG}-\mathrm{in})$.

During the operation of the Tevatron, the closed orbit has been "smoothed" at all energies. Beam positions were corrected to within $+/-.5 \mathrm{~mm}$ of the design orbit using the correction element dipoles. By examining the steering strengths necessary to smooth the orbit and working backward [using Eq. (3)], a linear approximation to the uncorrected Tevatron closed orbit may be obtained.

Figure 2(a) shows the smoothed Tevatron vertical orbit at $600 \mathrm{GeV}$. The horlzontal axts represents the four-mile circumference of the machine while the vertical axis shows the vertical displacements from the design orbit in millimeters. Figure $2(b)$ shows the strengths of the steering dipoles (in milliradians) which created the smooth orbit of Figure 2(a). Figure 2 (c) shows the calculated uncorrected Tevatron vertical orbit. The uncorrected closed orbit exhibits a typical displacement of

$$
\text { Xrms }=12.6 \mathrm{~mm} \quad \text { (calculated) }
$$

with a maximum of $33 \mathrm{~mm}$. These values ayree extremely well with those obtained in the design study. The steering dipole strengths also agree with the design yielding values of

$$
\begin{aligned}
& \theta_{\text {rms }}=32 \mu \mathrm{rad}, \\
& \theta_{\max }=110 \mu \mathrm{rad} .
\end{aligned}
$$

(observed)

Results from the horizontal correction dipoles yield similar results, though one must take into account an offset in the beam momentum. When the Tevatron horizontal orbit was corrected during commissioning, the horizontal correction dipoles corrected a 


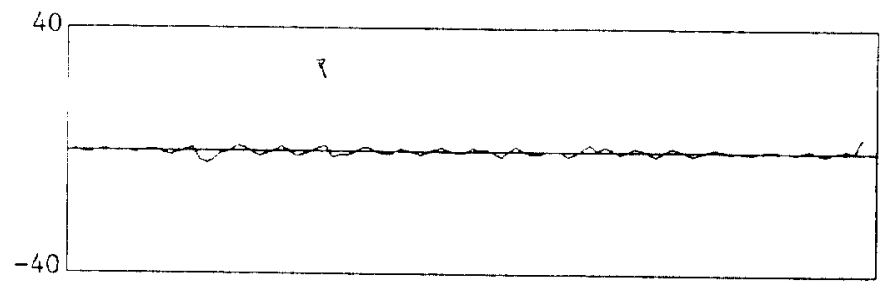

(a) Original Positions (mu)

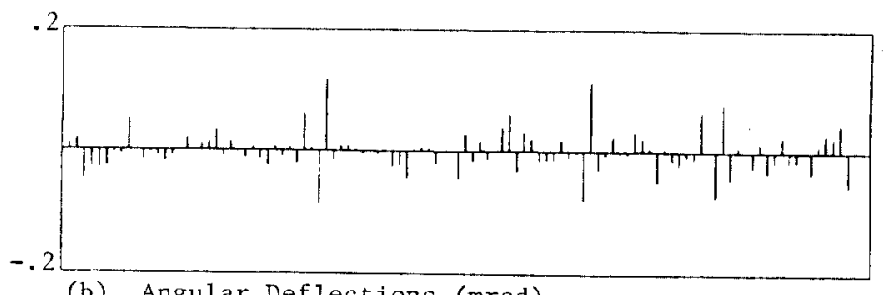

(b) Angular Deflections (mrad)

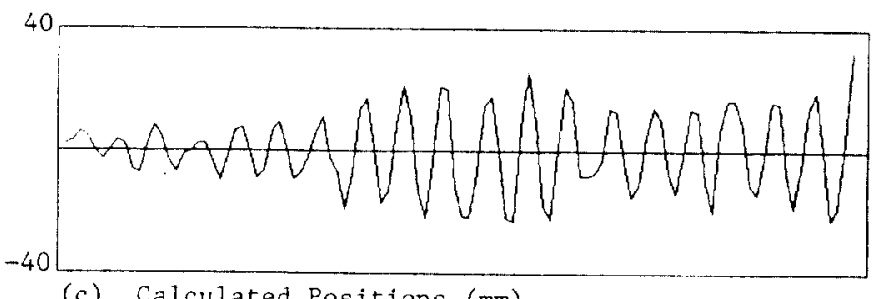

(c) Calculated Positions (mn)

Figure 2 (a) Tevatron $600 \mathrm{GeV}$ smoothed vertical orbit. (b) Correction element dipole strengths necessary to produce the orbit in (a). (c) The calculated $600 \mathrm{CeV}$ uncorrected vertical orbit.

mismatch between the guide field and the beam momentum. This resulted in a 32 urad average offset in the steering strengths of the correction dipoles after smoothing. This posed no apparent problem during the following $800 \mathrm{GeV}$ HEP run, yet must be corrected before I TeV operation.

\section{Extension to SSC Lattices}

The expressions developed earlier do not take into account orbit distortions generated by ficld errors in a low-beta insertion. However, they should still prove useful if the low-beta regions are designed with their own special orbit correction systems. The field strengths needed for the correction dipoles in the arcs of the accelerator may then be computed using Eq.'s (6) and (7).
Using SSC Test Lattice parameters $\left[\beta_{O}=340 \mathrm{~m}\right.$, $\langle B\rangle=220 \mathrm{~m}, F=100 \mathrm{~m}, \nu=85.35, \mu_{O}=60^{\circ}, N_{Q}=820$, $\mathrm{N}_{\mathrm{B}}=6600, \mathrm{n}_{\mathrm{Q}}=2, \mathrm{n}_{\mathrm{B}}=16(6.0$ [' magnets $\left.)\right]$, the expressions become

$$
\begin{aligned}
& \text { Xrms }=31 \mathrm{~mm} \quad\left[\mathrm{q}^{2}+1 / 14 \mathrm{~b}^{2}\right]^{\frac{1}{2}} \\
& \Theta_{\mathrm{rms}}=13 \mu \mathrm{rad}\left[\mathrm{q}^{2}+1 / 14 \mathrm{~b}^{2}\right]^{\frac{1}{2}} .
\end{aligned}
$$

Due to the weaker focusing properties of the SSC, the quadrupole alignment term appears to be even more dominant in the SSC than in the Tevatron. By using the same values of $b$ and $q$ in the above cxpressions as for the Tevatron, the expected rms orbit and steering strength become

and hence,

$$
\begin{aligned}
& \text { Xrms }=20 \mathrm{~mm} \\
& \theta_{\text {rms }}=8 \mathrm{\mu rad}, \\
& \theta_{\max }=25 \mathrm{\mu rad} .
\end{aligned}
$$

This would indicate that the SSC steering dipoles need to be capable of delivering an integrated field strength of at least $1.7 \mathrm{~T}-\mathrm{m}(660 \mathrm{kG}-\mathrm{in})$ at $20 \mathrm{TeV}$. To ensure orbit manipulations beyond centering, a field strength of $2.3 \mathrm{~T}-\mathrm{m}$ ( $880 \mathrm{kG}-\mathrm{in}$ ) would be recommended. of course, if quadrupole misalignments and/or field errors are greater than for the Tevatron, these numbers will be higher. For example, if $q=1, b=2$, the results become $\mathrm{Xrms}=35 \mathrm{~mm}, \theta_{\mathrm{rms}}=15 \mu \mathrm{rad}, \theta_{\mathrm{max}}=45 \mu \mathrm{rad}$ $(C \mathrm{Bd} \ell=3 \mathrm{~T}-\mathrm{m}=1200 \mathrm{kG}-\mathrm{in})$.

\section{Cuncluding Remarks}

The general expressions for the rms closed orbit distrotion $[\mathrm{Eq}$. (6)] and the rms dipole correction element steering strength [Eq. (7)] accurately describe the properties of the operational Tevatron. Extending these expressions to the SSC should provide an initial. feel for the types of orbit distortions to expect and the necessary field strengths of the correction dipoles through the major bending arcs of the accelerator.

\section{Bibliography}

1. Courant, E.D., and H.S. Snyder, "Theory of the Alternating Gradient Synchrotron," Annals of Physics, Vol. 3, No. 1, p. 18, January, 1958.

2. Bovet, C. et al, "A Selection of Formulae and Data Useful for the Design of A.G. Synchrotrons." Geneva, Switzerland: European Center for Nuclear Research. p. 24, Apri1, 1970.

3. "A Report on the Design of the Fermi National Accelerator Laboratory Superconducting Accelerator". Fermi National Accelerator Laboratory. p. 123, May, 1979. 